\title{
Molecular dynamics simulation of aqueous solutions of trimethylamine- $N$-oxide and tert-butyl alcohol
}

\author{
Arianna Fornili, ${ }^{a}$ Monica Civera, ${ }^{a}$ Maurizio Sironi ${ }^{a}$ and Sandro L. Fornili*b \\ a CISI and Department of Physical Chemistry and Electrochemistry, University of Milan, \\ Via Golgi 19, I-20133, Milan, Italy \\ ${ }^{b}$ INFM and Department of Information Technologies, University of Milan, Via Bramante 65, \\ I-26013, Crema, Italy. E-mail: fornili@dti.unimi.it; Fax: +3902 50330010
}

Received 18th July 2003, Accepted 4th September 2003

First published as an Advance Article on the web 26th September 2003

In this work we have investigated hydration properties of aqueous solutions up to a solute molar fraction $X_{2}=0.125$ of two isosteric molecules - the bioprotectant trimethylamine- $N$-oxide (TMAO) and the denaturant tert-butyl alcohol (TBA) - using molecular dynamics simulation at $298 \mathrm{~K}$. Statistical analyses of the trajectories show in particular that as the solute concentration increases the number of the water molecules in the first hydration shell decreases uniformly for TMAO, while for TBA it decreases more rapidly in a concentration range where experiments indicate that TBA starts to self-aggregate. No appreciable solute segregation occurs for TMAO even in the most concentrated solution, where on the average each water molecule is shared by two solutes. This result parallels what has been recently found for glycine betaine, an organic osmolyte closely related to TMAO.

\section{Introduction}

Trimethylamine- $N$-oxide (TMAO, Fig. 1 ) is a naturally occurring organic osmolyte, which counteracts the denaturing effects of urea. ${ }^{1,2}$ Moreover, experiments show that it behaves as a chemical chaperone, inducing the renaturation of proteins, ${ }^{3}$ while unaffecting their dynamics. ${ }^{4}$ Such behavior stems from the osmophobic effect consisting in an unfavorable interaction of osmolytes with the protein backbone, a newly recognized renaturing interaction which is particularly effective in a crowded environment such as the cell interior. ${ }^{5}$ This effect appears to be related to the osmolyte property of being displaced from the protein domain, ${ }^{6}$ which has been recently confirmed at microscopic level of details for the disaccharide trehalose using molecular dynamics (MD) simulation. ${ }^{7}$ The osmophobic effect appears also related to the osmolyte-water interaction. ${ }^{8}$

The hydration characteristics of TMAO up to a solute molar fraction $X_{2}=0.05$ have been experimentally compared with those of the isosteric molecule tert-butyl alcohol (TBA, Fig. 1), ${ }^{9,10}$ a particularly effective protein denaturant, featuring the largest hydrophobic moiety among the simple water-soluble alcohols. ${ }^{9}$ Results indicate that while TBA starts to self-aggre-
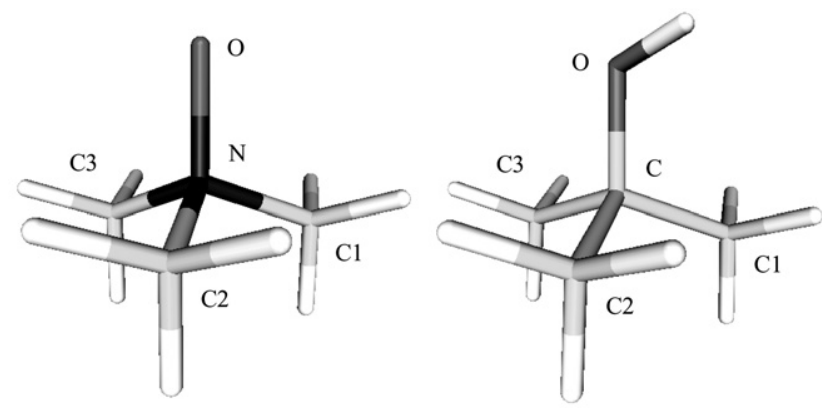

Fig. 1 TMAO (left) and TBA (right). gate at $c a . X_{2}=0.025$, TMAO does not exhibit any significant self-aggregation up to the highest concentration considered.

Microscopic details of the water interaction with glycine betaine ( $N, N, N$-trimethylglycine, GB), a molecule closely related to TMAO, have been recently investigated using MD in a wide range of solute concentration (i.e., 0.002-0.22 solute molar fraction). ${ }^{11}$ Statistical analyses of GB trajectories have shown that a diffuse intermixing of GB and water persists up to the most concentrated solution, where a water molecule is on the average shared by three GB molecules.

The same approach has been adopted in this work, which has been undertaken to investigate properties of aqueous solutions of TMAO and TBA in the $0.002-0.125$ solute molar fraction range.

\section{Computational methods}

Classical and QM MD simulations were performed using SANDER and ROAR modules of Amber $6,{ }^{12}$ respectively, with 0.5 fs time-step. For the QM simulations, the temperature was controlled according to the Nosé-Hoover scheme ${ }^{13}$ with a three-thermostats chain, while for the classical simulations the Berendsen coupling algorithm ${ }^{13}$ was used with $\tau_{\mathrm{t}}=1 \mathrm{ps}$; $\tau_{\mathrm{p}}=0.2 \mathrm{ps}$ was chosen for pressure control. No cutoff for $\mathrm{QM}$ atoms, while a $9 \AA$ cutoff and Ewald sums for classical nonbonded interactions were used. SHAKE ${ }^{13}$ kept constrained bonds involving hydrogen atoms. The TIP3P water model was assumed. ${ }^{14} \mathrm{Ab}$ initio calculations were done at the RHF/6$31++\mathrm{G}^{* *}$ level using PC-GAMESS. ${ }^{15,16}$ AM1-SRP parameters ${ }^{17,18}$ were optimized by using a nonlinear least-squares $\operatorname{code}^{19}$ which minimizes a penalty function involving relevant geometric parameters and torsional energy barriers.

\section{Results and discussion}

Following ab initio quantum mechanical full optimization of the TMAO and TBA molecular geometries, we evaluated their 
atomic charges according to the RESP procedure. ${ }^{20}$ Atomic charges and coordinates are reported in Tables 1 and 2 .

To properly describe the internal dynamics of TMAO and TBA, relevant parameters of the AMBER force field ${ }^{21,22}$ have been checked using the same approach previously employed for GB. ${ }^{11}$ Firstly, we evaluated with $a b$ initio quantum mechanical calculations the energy barrier to the rotation about the $\mathrm{C}-\mathrm{N}$ bond of TMAO and those about the bonds $\mathrm{C}-\mathrm{O}$ and $\mathrm{C}_{x}-\mathrm{C}$ of TBA, where $\mathrm{C}_{x}$ represents a methyl carbon atom. Then, so-called "AM1 specific reaction parameters" (AM1-SRP) ${ }^{17,18}$ were determined, to improve the description of TMAO and TBA by the AM1 hamiltonian. ${ }^{23}$ Indeed, by adopting AM1-SRP rather than the default AM1 parameters, the description of the torsional energy barriers improves from $35 \%$ to $72 \%$ of the ab initio values for TMAO, and from $29 \%$ and $56 \%$ to $60 \%$ and $80 \%$, respectively, for the above mentioned TBA energy barriers. The molecular geometries were left practically unaffected. The AM1-SRP values are reported together with the default AM1 parameters in Table 3. Finally, we performed in-vacuo 500 ps QM MD simulations based on the AM1 hamiltonian including the AM1-SRP parameters and MM MD simulations based on the Amber force field. Comparison of the time evolutions of dihedral angles over the QM and MM trajectories indicated that it was necessary to add a new oxygen type into the Amber force field for TMAO and to modify a few dihedral parameters for TBA in order to get a closer resemblance between QM and MM findings. Such parameter values are reported in Table 4.

The appropriate Lennard-Jones parameters present in the Amber force-field were adopted to model the nonbonded interactions of all TMAO and TBA atoms but the TMAO oxygen atom. For the latter, a change was done in order to get a better agreement between the TMAO oxygen-water oxygen radial distribution functions (RDFs), $g_{\mathrm{OO}}(r)$, averaged over a $3 \times 10^{7}$-move Metropolis Monte Carlo simulation based on previously calculated $a b$ initio atom-atom pair potentials ${ }^{24}$ and 500-ps NVT MM MD simulations of a system consisting of one TMAO and 504 water molecules. Comparison of the RDFs indicates that the parameters of the TIP3P water oxygen atom (i.e., the oxygen type $\mathrm{OW}$ ) yield the best agreement for the positions of the $g_{\mathrm{OO}}(\mathrm{r})$ first-peak maximum.

Then, NPT MD simulations $(T=298 \mathrm{~K}, P=1$ bar $)$ have been performed for the systems listed in Table 5, each one including 504 TIP3P water molecules and the indicated number of TMAO or TBA molecules. The length of the production phase of each TMAO simulation was 500 ps following a 50 ps equilibration phase, while each TBA simulation involved 1000 ps for the production phase after a 500 ps equilibration phase, due to the presence of larger fluctuations.

Table 1 Atomic coordinates (in $\AA$ ) and charges (in $e$ ) for TMAO

\begin{tabular}{lrrrr}
\hline Atom & \multicolumn{1}{l}{$y$} & \multicolumn{1}{l}{$z$} & \multicolumn{1}{c}{$q$} \\
\hline $\mathrm{O}$ & 0.0000 & 0.0000 & 1.4338 & -0.6367 \\
$\mathrm{~N}$ & 0.0000 & 0.0000 & 0.0655 & 0.2740 \\
$\mathrm{C} 1$ & 0.0000 & 1.3967 & -0.4107 & -0.2634 \\
$\mathrm{H} 11$ & 0.0000 & 1.4448 & -1.4925 & 0.1281 \\
$\mathrm{H} 12$ & -0.8793 & 1.8753 & -0.0099 & 0.1281 \\
$\mathrm{H} 13$ & 0.8793 & 1.8753 & -0.0099 & 0.1281 \\
$\mathrm{C} 2$ & 1.2096 & -0.6983 & -0.4107 & -0.2634 \\
$\mathrm{H} 21$ & 1.2512 & -0.7224 & -1.4925 & 0.1281 \\
$\mathrm{H} 22$ & 2.0638 & -0.1761 & -0.0099 & 0.1281 \\
$\mathrm{H} 23$ & 1.1844 & -1.6992 & -0.0099 & 0.1281 \\
$\mathrm{C} 3$ & -1.2096 & -0.6983 & -0.4107 & -0.2634 \\
$\mathrm{H} 31$ & -1.2512 & -0.7224 & -1.4925 & 0.1281 \\
$\mathrm{H} 32$ & -1.1844 & -1.6992 & -0.0099 & 0.1281 \\
$\mathrm{H} 33$ & -2.0638 & -0.1761 & -0.0099 & 0.1281 \\
\hline
\end{tabular}

Table 2 Atomic coordinates (in $\AA$ ) and charges (in $e$ ) for TBA

\begin{tabular}{lrrrr}
\hline Atom & \multicolumn{1}{l}{$y$} & \multicolumn{1}{l}{$z$} & \multicolumn{1}{l}{$q$} \\
\hline H & 0.9332 & 0.0003 & 1.7242 & 0.4228 \\
O & 0.0305 & -0.0004 & 1.4350 & -0.7425 \\
C & -0.0050 & 0.0000 & 0.0189 & 0.6338 \\
C1 & -1.4863 & -0.0047 & -0.3420 & -0.3231 \\
H11 & -1.6238 & -0.0047 & -1.4183 & 0.0728 \\
H12 & -1.9692 & -0.8848 & 0.0685 & 0.0728 \\
H13 & -1.9749 & 0.8720 & 0.0691 & 0.0728 \\
C2 & 0.6782 & 1.2621 & -0.5109 & -0.3231 \\
H21 & 0.6333 & 1.3121 & -1.5939 & 0.0728 \\
H22 & 0.2005 & 2.1469 & -0.1042 & 0.0728 \\
H23 & 1.7265 & 1.2831 & -0.2236 & 0.0728 \\
C3 & 0.6864 & -1.2571 & -0.5120 & -0.3231 \\
H31 & 0.6421 & -1.3064 & -1.5950 & 0.0728 \\
H32 & 1.7348 & -1.2716 & -0.2245 & 0.0728 \\
H33 & 0.2144 & -2.1454 & -0.1062 & 0.0728 \\
\hline
\end{tabular}

Structural and dynamic properties of the water surrounding the solutes have been examined by evaluating the average numbers of water molecules belonging to the first hydration shell of solutes and their self-diffusion coefficient values, $D$. They are reported in Table 5 together with the number of the water molecules hydrogen-bonded to the oxygen atom of TMAO or to the TBA hydroxyl group. A water molecule is assumed to belong to the first hydration shell if the distance of its oxygen atom from the solute atoms is less than or equal to $3.5 \AA$. We also assume that a water molecule is hydrogenbonded if the oxygen-oxygen distance is $\mathrm{d}_{\mathrm{OO}} \leq 3.5 \AA$ and the oxygen-hydrogen-oxygen angle is $\theta_{\mathrm{OHO}} \geq 150^{\circ} .{ }^{25}$ The water diffusion coefficient $D$ is evaluated according to the Einstein equation: ${ }^{13}$

$$
\left\langle\left|\boldsymbol{r}_{i}(t)-\boldsymbol{r}_{i}(0)\right|^{2}\right\rangle=6 D t
$$

whose left-hand side represents the mean square displacement of the first-shell waters as a function of time, being $\boldsymbol{r}_{i}(t)$ and $\boldsymbol{r}_{i}(0)$ their positions at time $t$ and at the start of each of the segments in which the trajectories are subdivided, respectively. A 1.5-ps segment duration was found a reasonable compromise between the contrasting requirements of being short enough to monitor the mobility of waters within the first-shell space region, and long enough to make the Einstein equation applicable. ${ }^{26}$ For comparison, in Table 5 we also report the $D$ value for TIP3P water obtained analyzing the trajectory of a 500 ps NPT MD simulation at $300 \mathrm{~K}$ of a system consisting of 454 water molecules, one of which is taken as "solute".

Table 3 AM1 default and "specific reaction parameters" (SRP) for TMAO and TBA

\begin{tabular}{llrrr}
\hline Atom & Parameter $^{a}$ & \multicolumn{1}{l}{ AM1 } & SRP (TMAO) & SRP (TBA) \\
\hline $\mathrm{H}$ & $\zeta_{\mathrm{s}}$ & 1.188078 & 1.443226 & 1.416569 \\
$\mathrm{H}$ & $\alpha$ & 2.882324 & 2.510250 & 2.490328 \\
$\mathrm{C}$ & $U_{\mathrm{pp}}$ & -39.614239 & -38.511379 & -38.726880 \\
$\mathrm{C}$ & $\beta_{\mathrm{s}}$ & -15.715783 & -23.573675 & -17.978856 \\
$\mathrm{C}$ & $\beta_{\mathrm{p}}$ & -7.719283 & -11.578925 & -10.807367 \\
$\mathrm{C}$ & $\zeta_{\mathrm{s}}$ & 1.808665 & 1.483105 & 1.583978 \\
$\mathrm{C}$ & $\zeta_{\mathrm{p}}$ & 1.685116 & 1.561091 & 1.978191 \\
$\mathrm{~N}$ & $\zeta_{\mathrm{s}}$ & 2.315410 & 2.400000 & - \\
$\mathrm{N}$ & $\zeta_{\mathrm{p}}$ & 2.157940 & 2.149108 & - \\
$\mathrm{N}$ & $G_{\mathrm{p} 2}$ & 11.59 & 10.778700 & - \\
$\mathrm{O}$ & $\beta_{\mathrm{s}}$ & -29.272773 & -29.837152 & -31.478769 \\
$\mathrm{O}$ & $\beta_{\mathrm{p}}$ & -29.272773 & -29.837152 & -31.478769 \\
$\mathrm{O}$ & $\zeta_{\mathrm{p}}$ & 2.524039 & 2.477462 & 2.701768
\end{tabular}

${ }^{a} \zeta$, Slater exponents; $\alpha$, core-core repulsion integral; $\beta$, resonance integral; $U_{\mathrm{pp}}$, one-center core-electron attraction + kinetic energy; $G_{\mathrm{p} 2}$, one-center electron repulsion integral. 
Table 4 Modified TMAO and TBA force field torsional parameters listed according to the AMBER format. ${ }^{21}$ The Lennard-Jones parameters of the new oxygen atom type OF are the same as for the TIP3P water oxygen type, OW

\begin{tabular}{llllr}
\hline Torsion & $m^{a}$ & $V_{n} / 2^{b}$ & $\gamma^{c}$ & $n^{d}$ \\
\hline X-CT-N3-OF (TMAO) & 9 & 0.0675 & 0.0 & 3 \\
HO-OH-CT-CT (TBA) & 1 & 0.2 & 0.0 & -3 \\
HO-OH-CT-CT (TBA) & 1 & 0.25 & 0.0 & 1 \\
HC-CT-CT-OH (TBA) & 1 & 0.0 & 0.0 & 1
\end{tabular}

0.0

0.0

${ }^{a}$ Number of bond paths; ${ }^{b}$ barrier height (in $\mathrm{kcal} / \mathrm{mol}$ ) divided by two; ${ }^{c}$ phase shift in degrees; ${ }^{d}$ periodicity of the torsional barrier.

From Table 5 one can see that as the solute concentration increases the average number of the first-shell waters decreases, as expected, due to the mutual interference of the hydration shells surrounding the individual solute molecules. However, the different behaviors of TMAO and TBA become evident by comparing the most concentrated solutions with the least concentrated ones: the number of the first-shell waters decreases by $22 \%$ for TMAO and by $55 \%$ for TBA. It is worth noting that a $22 \%$ decrease in the number of first-shell waters was also found for GB between the one-solute and 64-solute systems involving 453 water molecules, ${ }^{11}$ namely for a similar increase of solute molar fraction.

A more detailed picture is provided by Fig. 2, which shows that the average number of the first-shell waters decreases uniformly for TMAO while a more complex behavior is present for TBA. In particular, the latter exhibits a rather steep decrease in the $0.02-0.03$ solute molar fraction range, that is in the same concentration range where remarkable changes in a number of physical properties have been experimentally shown to occur for aqueous solutions of TBA but not for those of TMAO. ${ }^{10}$ These experimental findings were interpreted as

Table 5 Average number per solute of water molecules within $3.5 \AA$ from the solute atoms (First-shell waters) and their self-diffusion coefficient, $D$ (in $10^{-5} \mathrm{~cm}^{2} \mathrm{~s}^{-1}$ ) [some of them are hydrogen-bonded to solute $(H-B$ waters $)]$. Left and right numbers pertain to TMAO and TBA, respectively

\begin{tabular}{|c|c|c|c|c|c|c|c|}
\hline \multirow{2}{*}{$\begin{array}{c}\text { Solutes } \\
1\end{array}$} & \multirow{2}{*}{$\begin{array}{l}\begin{array}{l}\text { Solute } \\
\text { molar } \\
\text { fraction }\end{array} \\
1.98 \times 10^{-3}\end{array}$} & \multicolumn{2}{|c|}{$\begin{array}{l}\text { First-shell } \\
\text { waters }\end{array}$} & \multicolumn{2}{|c|}{ H-B waters } & \multicolumn{2}{|c|}{$D$} \\
\hline & & 17.2 & 16.5 & 2.5 & 1.3 & 4.5 & 5.0 \\
\hline 4 & $7.87 \times 10^{-3}$ & 17.0 & 16.2 & 2.4 & 1.3 & 4.4 & 4.6 \\
\hline 6 & $1.18 \times 10^{-2}$ & 16.7 & 15.6 & 2.4 & 1.3 & 4.0 & 4.3 \\
\hline 8 & $1.56 \times 10^{-2}$ & 16.9 & 15.1 & 2.4 & 1.3 & 4.0 & 4.1 \\
\hline 10 & $1.95 \times 10^{-2}$ & 16.7 & 15.1 & 2.4 & 1.3 & 3.8 & 4.1 \\
\hline 12 & $2.33 \times 10^{-2}$ & 16.7 & 15.0 & 2.4 & 1.3 & 3.6 & 3.9 \\
\hline 14 & $2.70 \times 10^{-2}$ & 16.5 & 14.4 & 2.4 & 1.3 & 3.4 & 3.7 \\
\hline 16 & $3.08 \times 10^{-2}$ & 16.4 & 12.2 & 2.4 & 1.2 & 3.4 & 3.5 \\
\hline 18 & $3.45 \times 10^{-2}$ & 16.2 & 11.9 & 2.4 & 1.2 & 3.2 & 3.5 \\
\hline 20 & $3.82 \times 10^{-2}$ & 16.3 & 11.6 & 2.4 & 1.2 & 3.1 & 3.4 \\
\hline 22 & $4.18 \times 10^{-2}$ & 16.2 & 11.9 & 2.4 & 1.2 & 2.9 & 3.3 \\
\hline 24 & $4.55 \times 10^{-2}$ & 16.0 & 11.0 & 2.4 & 1.2 & 2.8 & 3.1 \\
\hline 26 & $4.91 \times 10^{-2}$ & 15.8 & 11.7 & 2.4 & 1.2 & 2.7 & 3.1 \\
\hline 28 & $5.26 \times 10^{-2}$ & 15.9 & 9.9 & 2.4 & 1.1 & 2.6 & 3.0 \\
\hline 30 & $5.62 \times 10^{-2}$ & 15.7 & 10.7 & 2.4 & 1.2 & 2.5 & 2.9 \\
\hline 34 & $6.32 \times 10^{-2}$ & 15.5 & 10.0 & 2.4 & 1.1 & 2.3 & 2.8 \\
\hline 38 & $7.01 \times 10^{-2}$ & 15.3 & 9.0 & 2.4 & 1.1 & 2.1 & 2.6 \\
\hline 42 & $7.69 \times 10^{-2}$ & 15.1 & 8.9 & 2.3 & 1.1 & 2.0 & 2.4 \\
\hline 47 & $8.53 \times 10^{-2}$ & 14.9 & 8.8 & 2.3 & 1.1 & 1.8 & 2.1 \\
\hline 53 & $9.52 \times 10^{-2}$ & 14.7 & 8.0 & 2.3 & 1.0 & 1.7 & 1.8 \\
\hline 58 & $1.03 \times 10^{-1}$ & 14.4 & 8.1 & 2.3 & 1.1 & 1.6 & 1.6 \\
\hline 64 & $1.13 \times 10^{-1}$ & 14.0 & 7.8 & 2.2 & 1.1 & 1.5 & 1.5 \\
\hline 72 & $1.25 \times 10^{-1}$ & 13.4 & 7.3 & 2.2 & 1.0 & 1.5 & 1.4 \\
\hline TIP3P water & & 7.3 & & 2.7 & & 5.9 & \\
\hline
\end{tabular}

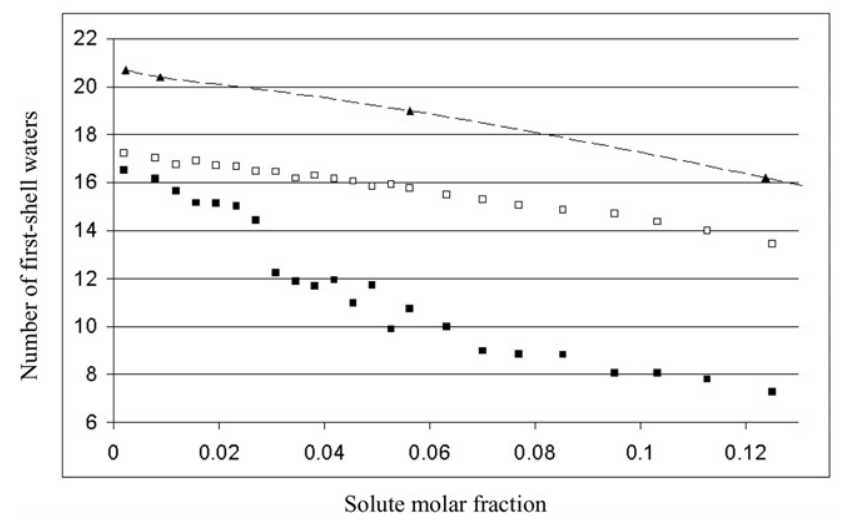

Fig. 2 Average number of water molecules residing within the first hydration shell of TMAO (open squares) and TBA (full squares) vs. solute molar fraction. GB data (triangles) are taken from ref. 11.

indicating that in the above concentration range TBA starts to self-aggregate while TMAO apparently does not selfaggregate up to the highest concentration considered (i.e., $\left.X_{2}=0.05\right) .{ }^{9}$ Moreover, analogous data for aqueous solutions of GB represented in Fig. 2, which were taken from ref. 11, indicate that the interaction with water of TMAO is similar to that of GB.

Data plotted in Fig. 3 are consistent with the above view. Indeed, the difference between the fractions of the water molecules not belonging to the first hydration shell of TBA and TMAO increases with increasing solute concentration. This suggests that while an extended intermixing of TMAO molecules and water persists up to the highest concentration, as it was found for $\mathrm{GB},{ }^{11}$ the solute segregation from solvent becomes more and more relevant in TBA solutions. Such a different behavior is pictorially represented in Fig. 4, where we show statistically representative snapshots taken from the trajectories of the most-concentrated solutions considered in this work.

In Fig. 5 we show the statistical distributions of the water surrounding TMAO and TBA for four solute molar fractions, namely $1.98 \times 10^{-3}, 2.70 \times 10^{-2}, 3.08 \times 10^{-2}$ and $1.25 \times 10^{-1}$. The second and third values fall in the concentration interval where a steep change occurs for TBA in the first-shell hydration number shown in Fig. 2. Again, the difference in the behavior of the two molecules increases with solute concentration. In particular, comparison of panels (a) and (c), where we report the probability that a given number of water molecules is present in the first-hydration shell of a solute, shows that the distributions relative to the second and third of the above concentration values are almost overlapping for TMAO while they

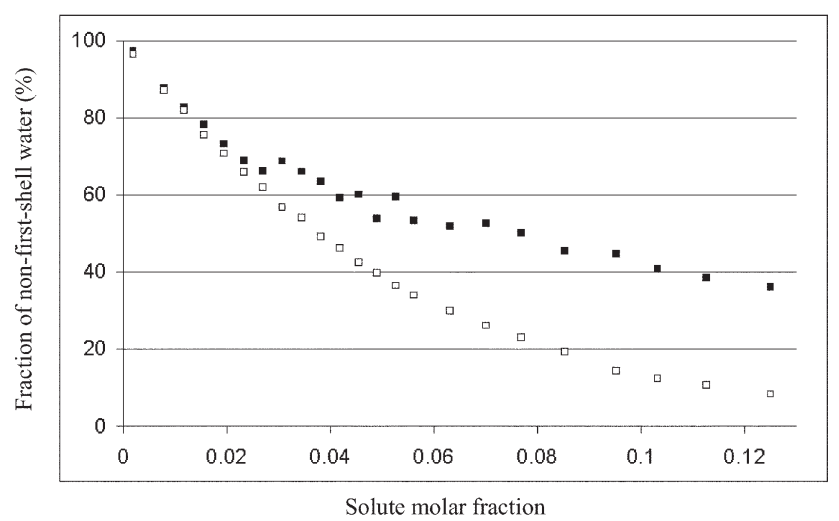

Fig. 3 Fraction of water not belonging to the first hydration shells of TMAO (open squares) and TBA (full squares) vs. solute molar fraction. 


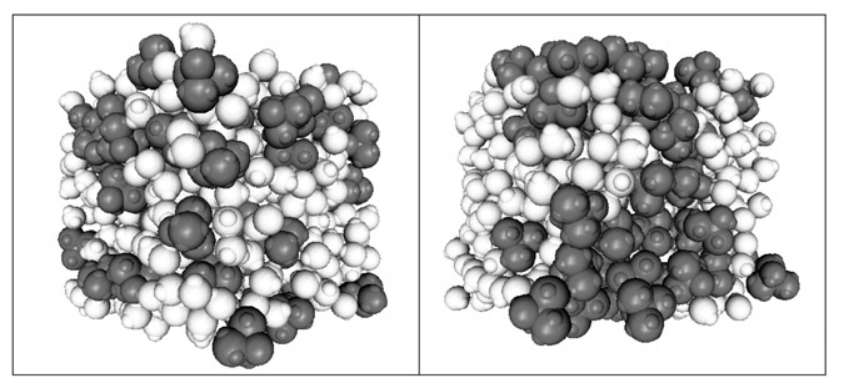

Fig. 4 Statistically representative snapshots of TMAO (left) and TBA (right) taken from the trajectories corresponding to the highest solute concentrations. Lighter molecules represent water.

are well separated for TBA. Moreover, in the most concentrated TBA solution the probability that no water molecules lies within a $3.5 \AA$ region surrounding a solute molecule is remarkable. Instead, this probability value corresponds to the presence of $c a$. nine water molecules in the first hydration shell of TMAO at the same concentration. Indeed, the probability that less than six waters surround a solute molecule vanishes even at the highest TMAO concentration considered. Analogous conclusions can be drawn from panels (b) and (d), where the probability is reported that a water molecule resides at the same time in the first hydration shell of a given number of solutes. Indeed, at the highest concentration considered, a water molecule is most probably shared by two TMAO molecules, while a water molecule most probably lies in the first shell of one TBA molecule. Again, the TMAO behaves like $\mathrm{GB}$, for which it was found that at $X_{2}=0.2$ one water molecule is most probably shared by three solute molecules. ${ }^{11}$

From these results, water sharing by solutes appears as a mechanism common to both TMAO and GB to preserve intermixing of solute and water molecules up to highest solute concentrations. This conclusion is even more evident in Fig. 6, where we represent the average number of solutes that share the same water molecule, as calculated on the basis of probability distributions of the kind shown in the panels (b) and (d) of Fig. 5, and analogous data for GB. ${ }^{11}$ Moreover,

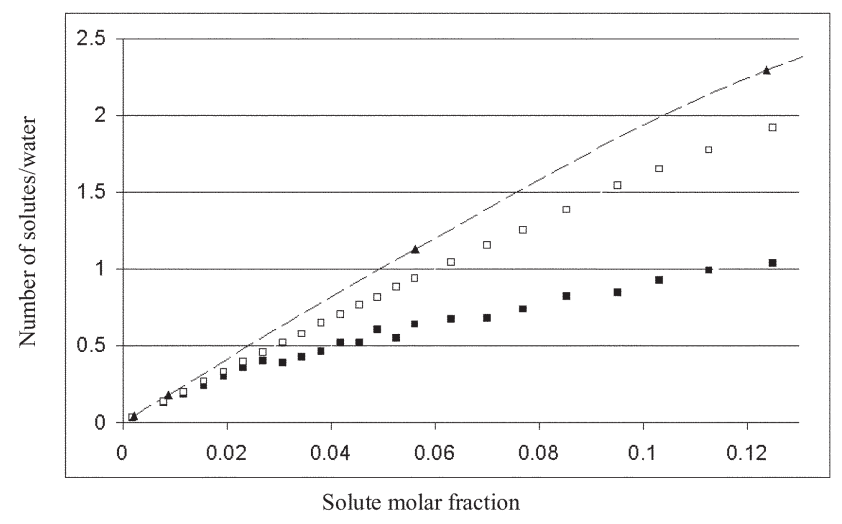

Fig. 6 Average number of solute molecules that share at the same time a water molecule in TMAO (open squares) and TBA (full squares) solutions vs. solute molar fraction. GB data (triangles) are taken from ref. 11 .

Fig. 6 indicates that the above mechanism is somewhat more effective for GB than for TMAO, possibly due to the presence in the former of two oxygen atoms. This result possibly parallels experimental findings showing that GB is more effectively excluded from a protein domain than TMAO. ${ }^{27}$

Oxygen-oxygen RDFs, $g_{\mathrm{oo}}(r)$, are reported in Fig. 7 for the most diluted and the most concentrated solutions considered: panels (a) and (c) show RDFs of water oxygen atoms around the oxygen atoms of TMAO and TBA solutions, respectively. Analogously, panels (b) and (d) report the water oxygenoxygen RDFs for TMAO and TBA, respectively. Panels (a) and (c) indicate that the water localization increases for both solute types as their concentration increases, but changes in first-peak height are somewhat larger for TBA than for TMAO. Water structuring is even more evidenced by the water RDFs of panels (b) and (d), where the second and third peaks become visible at the highest solute concentrations, while in the bulk TIP3P water the second RDF peak is barely detectable. ${ }^{14}$ Together with the above mentioned findings, and in particular with data shown in Fig. 2 and Fig. 3, these results indicate that TBA molecules keep self-aggregating as solute

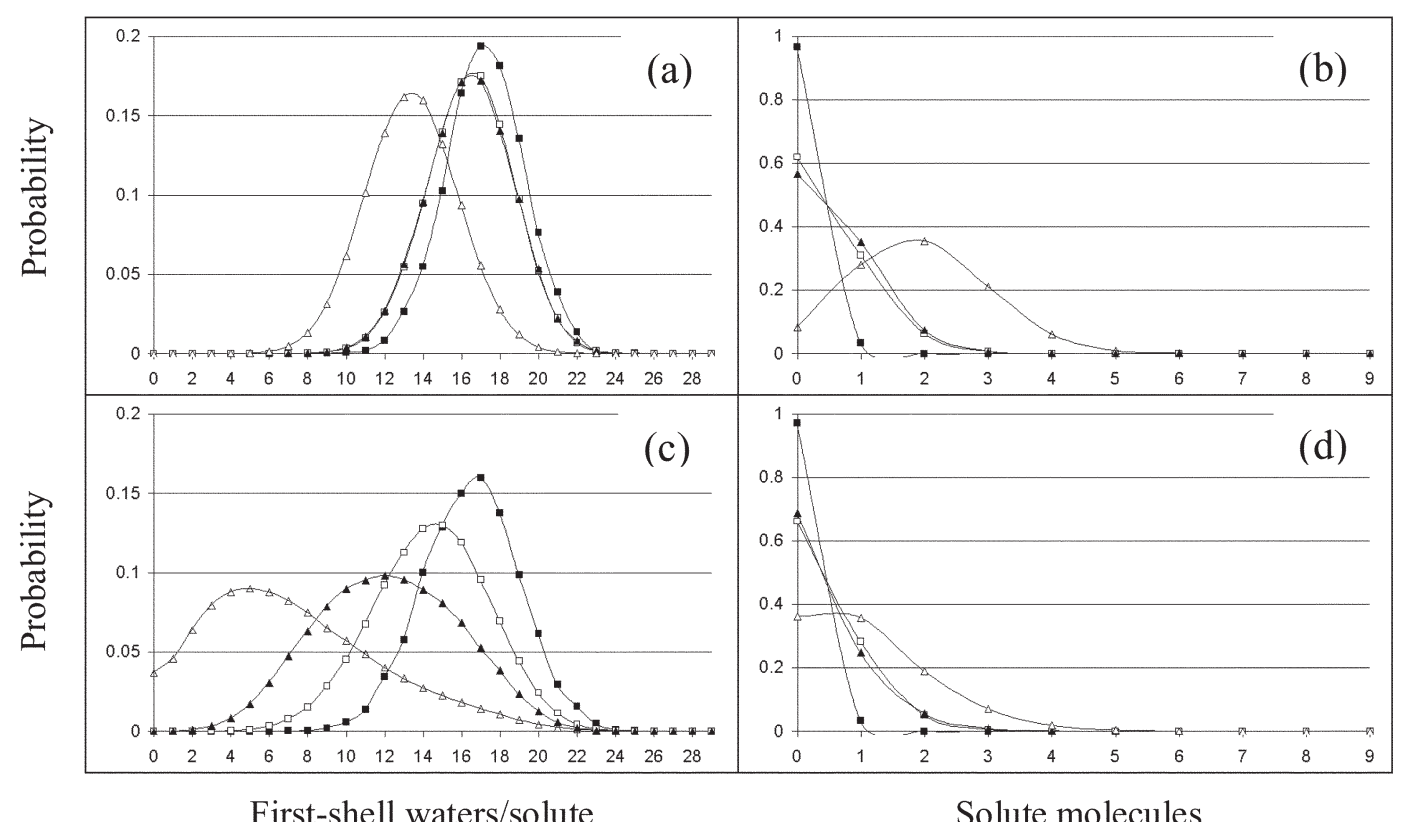

Fig. 5 Left: probability that a given number of water molecules are found within the first hydration shell of a solute. Right: probability that a water molecule resides at the same time in the first hydration shell of a given number of solutes. Panels (a) and (b) correspond to TMAO and panels $(\mathrm{c})$ and $(\mathrm{d})$ correspond to TBA. Full squares, open squares, full triangles and open triangles correspond to solute molar fractions $X_{2}=1.98 \times 10^{-3}, X_{2}=2.70 \times 10^{-2}, X_{2}=3.08 \times 10^{-2}$ and $X_{2}=1.25 \times 10^{-1}$, respectively. Lines are a guide for eyes. 


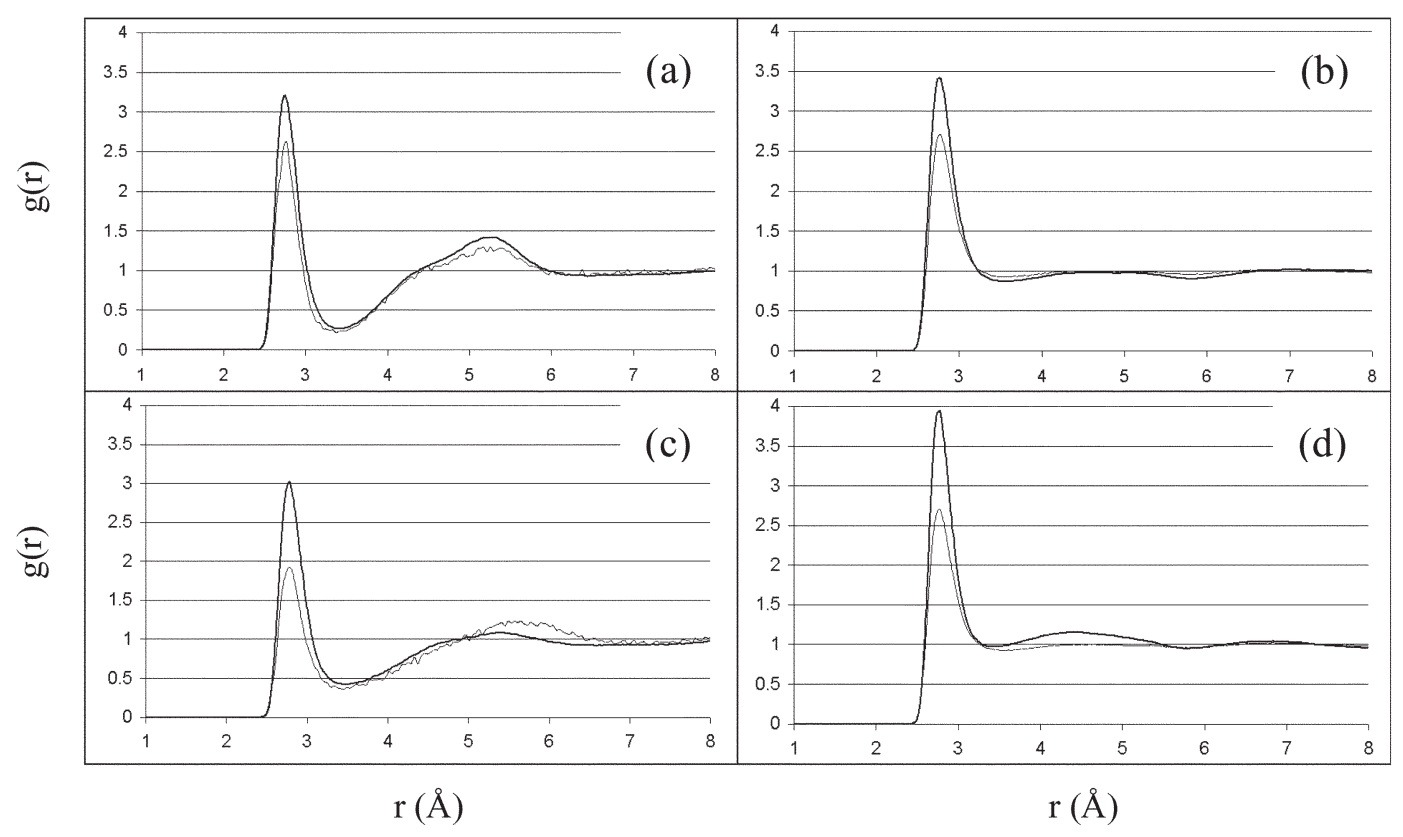

Fig. 7 Oxygen-oxygen radial distribution functions (RDFs), $g_{\mathrm{OO}}(r)$, for the lowest and highest solute concentration considered, i.e., $X_{2}=1.98 \times 10^{-3}$ and $X_{2}=1.25 \times 10^{-1}$. (a): TMAO oxygen-water oxygen; (b): water oxygen-water oxygen in TMAO solutions; (c): TBA oxygen-water oxygen; (d): water oxygen-water oxygen in TBA solutions. Light and heavy lines correspond to the lowest and the highest concentrations, respectively.

concentration increases and the water localization occurs for TBA at a higher rate than for the corresponding TMAO solutions. The latter behaves as GB: ${ }^{11}$ as the solute concentration increases more water molecules are shared between the hydrophilic moieties of the solute molecules, possibly bringing about some straining of the water-water hydrogen bonding. Structuring is, instead, apparently favored in the "water domain" of concentrated TBA solutions, as indicated by the first-peak maximum of the water oxygen-oxygen RDF corresponding to the most concentrated solution. This result agrees with recent experimental and simulation findings concerning a very concentrated aqueous solution of methanol, ${ }^{28}$ and with results of accurate simulations of moderately concentrated aqueous solutions of TBA, using different potentials and water model. ${ }^{29}$

Data reported in Table 5 for the self-diffusion coefficient of the first-shell water molecules, show that their translational dynamics is more restrained by TMAO than by TBA at all concentrations but the highest ones, where $D$ values become comparable. This result is consistent with previous $a b$ initio quantum molecular calculations showing that the interaction with water is stronger for TMAO than for TBA, ${ }^{24}$ and with recent findings obtained by simulation of aqueous solutions of TMAO based on a different force field. ${ }^{8}$

A three-dimensional visualization of the water distributions in the first coordination shells of TMAO and TBA is presented in Fig. 8 in terms of spatial distribution functions (SDFs) of the water oxygen atoms surrounding these solutes. Each SDF, evaluated for the corresponding aqueous solution of Table 5 that includes one solute molecule, provides an overall view of the way the solute interacts with water, from which its behavior in more concentrated solutions stems. For comparison, a GB SDF is also reported which is based on previous simulation data. ${ }^{11}$ Each SDF is calculated as follows: ${ }^{30}$

$$
g(\boldsymbol{r})=\rho(\boldsymbol{r}) / \rho_{0}
$$

where $\rho(\boldsymbol{r})$ indicates the number density of the water oxygen atoms at the position $\boldsymbol{r}$ and $\rho_{0}$ its average within the simulation box. The position vector $\boldsymbol{r}$ is defined in a coordinate system attached to the solute molecule. The isosurfaces reported in Fig. 8 correspond to $g(\boldsymbol{r})=3$. This figure shows that the water distribution around the solute hydrophobic moiety is markedly less evident for TBA than for TMAO, in agreement with previous results. ${ }^{24}$ Moreover, the SDF for the latter is larger between adjacent methyl groups, indicating that the presence of the nitrogen atom partially neutralizes the hydrophobic effects of these groups, thus disfavoring the self-aggregation at higher solute concentrations. This characteristic is even more evident in the GB SDF suggesting that it could be a common feature of the quaternary ammonium osmolytes.

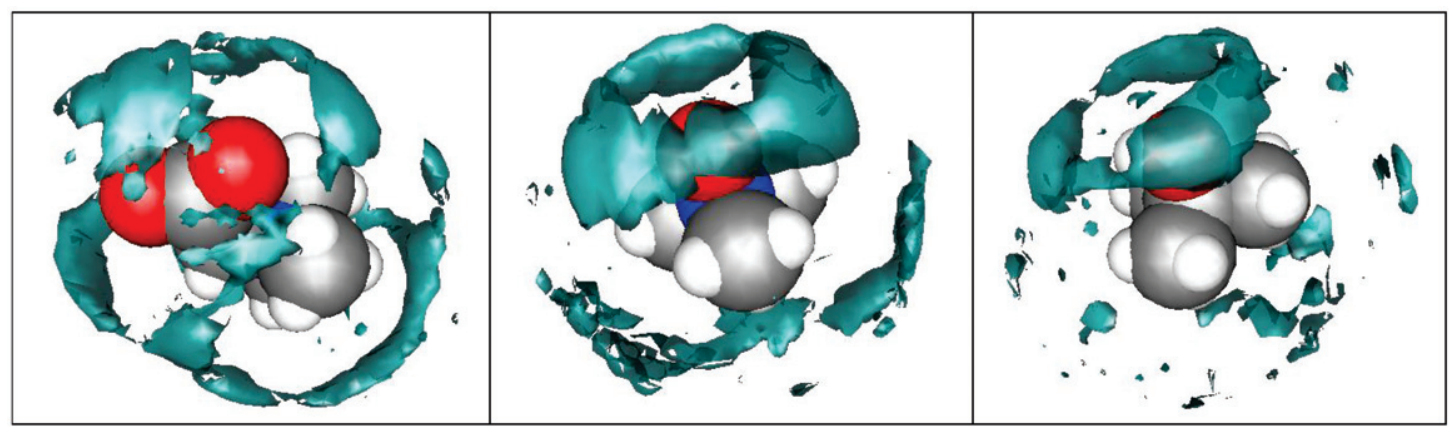

Fig. 8 Spatial distribution functions of the water oxygen atoms surrounding GB (left), TMAO (center) and TBA (right). The isosurfaces correspond to $g(\boldsymbol{r})=3$. 


\section{Conclusions}

MD simulation has been used to investigate properties of aqueous solutions of the isosteric molecules TMAO (a bioprotectant) and TBA (a protein denaturant) in a wide range of solute molar fractions. A preliminary fine-tuning of relevant forcefield parameters has been made by means of ab initio quantum mechanical calculations and QM MD simulations to guarantee a proper modeling of the solute internal dynamics.

Statistical analyses of the trajectories evidence behaviors of these solutes in quantitative agreement with experimental findings, ${ }^{9,10}$ indicating that TBA starts to self-aggregate in the same solute concentration range suggested by experiment, while TMAO does not appreciably self-aggregate up to the highest concentration considered. In particular, the analysis of the water distribution around the solutes indicates that in TMAO solutions a diffuse solute-solvent intermixing persists even in the most concentrated solution, where each water molecule is most probably shared by two solute molecules. The TMAO behavior is then similar to that recently shown for the closely related osmolyte $\mathrm{GB},{ }^{11}$ which, however, appears to exploit more effectively than TMAO this common strategy to extend solubility to the highest concentrations. This result possibly parallels previous experimental findings showing that GB is more strongly excluded by protein surface than TMAO. ${ }^{27}$

Solute segregation in TBA solutions is particularly evidenced by system snapshots of the most concentrated solution, for which the water distribution analysis indicates that a relevant fraction of water does not reside in the first hydration shell of solute molecules. Moreover, at this concentration the water oxygen-oxygen radial distribution function indicates that the water localization in TBA is tighter than in TMAO, suggesting that in concentrated TBA solutions the water structure is enhanced, in analogy with recent experimental and simulation findings for a very concentrated aqueous solution of methanol. ${ }^{28}$

\section{Acknowledgements}

We thank D. Rebeccani, G. P. Ghilardi and P. Ferri for keeping our Linux cluster alive. Support by FIRST funds is acknowledged.

\section{References}

1 P. H. Yancey, M. E. Clark, S. C. Hand, R. D. Bowlus and G. N. Somero, Science, 1982, 217, 1214.

2 P. H. Yancey, W. R. Blake and J. Conley, Comp. Biochem. Physiol. A, 2002, 133, 667.
3 C. M. Hill, I. R. Bates, G. F. White, F. R. Hallett and G. Harauz, J. Struct. Biol., 2002, 139, 13.

4 M. Gonnelli and G. B. Strambini, Biophys. Chem., 2001, 89, 77.

5 D. W. Bolen and I. V. Basakov, J. Mol. Biol., 2001, 310, 955.

6 T. Arakawa and S. N. Timasheff, Biophys. J., 1985, 47, 411.

7 G. Cottone, G. Ciccotti and L. Cordone, J. Chem. Phys., 2002, 117, 9862.

8 Q. Zou, B. J. Bennion, V. Daggett and K. P. Murphy, J. Am. Chem. Soc., 2002, 124, 1192.

9 M. Freda, G. Onori and A. Santucci, J. Phys. Chem. B, 2001, 105, 12714.

10 M. Freda, G. Onori and A. Santucci, Phys. Chem. Chem. Phys., 2002, 4, 4979

11 M. Civera, A. Fornili, M. Sironi and S. L. Fornili, Chem. Phys. Lett., 2003, 367, 238.

12 D. A. Case, D. A. Pearlman, J. W. Caldwell, T. E. Cheatam, III, W. S. Ross, C. L. Simmerling, T. A. Darden, K. M. Merz, R. V. Stanton, A. L. Cheng, J. J. Vincent, M. Crowley, V. Tsui, R. J. Radmer, Y. Duan, J. Pitera, I. Massova, G. L. Seibel, U. C. Singh, P. K. Weiner and P. A. Kollman, Amber 6, University of California, San Francisco, 1999

13 M. P. Allen and D. J. Tildesley, Computer Simulation of Liquids, Clarendon Press, Oxford, 1987.

14 W. L. Jorgensen, J. Chandrasekhar, J. D. Madura, R. W. Impey and M. L. Klein, J. Chem. Phys., 1983, 79, 926.

15 M. W. Schmidt, K. K. Baldridge, J. A. Boatz, S. T. Elbert, M. S. Gordon, J. H. Jensen, S. Koseki, N. Matsunaga, K. A. Nguyen, S. J. Su, T. L. Windus, M. Dupuis and J. A. Montgomery, J. Comput. Chem., 1993, 14, 1347.

16 A. A. Granovsky, http://classic.chem.msu.su/gran/gamess/ index.html.

17 A. Gonzalez-Lafont, T. N. Truong and D. G. Truhlar, J. Phys. Chem., 1991, 95, 4618.

18 Y. Kim, J. Am. Chem. Soc., 1996, 118, 1522.

19 G. Granucci and S. Inglese, private communication.

20 C. I. Bayly, P. Cieplak, W. D. Cornell and P. A. Kollman, J. Phys. Chem., 1993, 97, 10269

21 W. D. Cornell, P. Cieplak, C. I. Bayly, I. R. Gould, K. M. Merz, D. M. Ferguson, D. C. Spellmeyer, T. Fox, J. W. Caldwell and P. A. Kollman, J. Am. Chem. Soc., 1995, 117, 5179.

22 J. Wang, P. Cieplak and P. A. Kollman, J. Comput. Chem., 2000, 21, 1049.

23 M. J. S. Dewar, E. G. Zoebisch, E. F. Healy and J. J. P. Stewart, J. Am. Chem. Soc., 1985, 107, 3902.

24 R. Noto, V. Martorana, A. Emanuele and S. L. Fornili, J. Chem. Soc. Faraday Trans., 1995, 91, 3803.

25 H. Xu and B. J. Berne, J. Phys. Chem. B, 2001, 105, 11929.

26 G. Bonanno, R. Noto and S. L. Fornili, J. Chem. Soc. Faraday Trans., 1998, 94, 2755.

27 E. S. Courtenay, M. W. Capp, C. F. Anderson and M. T. Record, Jr., Biochemistry, 2000, 39, 4455.

28 S. Dixit, J. Crain, W. C. K. Poon, J. L. Finney and A. K. Soper, Nature, 2002, 416, 829.

29 P. G. Kusalik, A. P. Lyubartsev, D. L. Bergman and A. Laaksonen, J. Phys. Chem. B, 2000, 104, 9533.

30 I. M. Svishchev and P. G. Kusalik, J. Chem. Phys., 1993, 99, 3049. 